\title{
Reflectarray Antennas
}

\author{
Kshitij Lele \\ P.G. Student, \\ Department of EXTC \\ DJ Sanghvi College \\ of Engineering
}

\author{
Ami A. Desai \\ P.G. Student \\ Department of EXTC \\ DJ Sanghvi College \\ of Engineering
}

\author{
Ameya A. Kadam \\ Assistant Professor, \\ Department of EXTC \\ DJ Sanghvi College \\ of Engineering
}

\author{
Amit A. \\ Deshmukh \\ Professor and Head, \\ Department of EXTC, \\ DJ Sanghvi College \\ of Engineering
}

\begin{abstract}
Printed reflectarrays combine certain advantages of reflector antennas and phased arrays. One major hurdle in the designing of reflectarrays is the compensation of phase difference between various elements. This paper presents reviews of various techniques for phase compensation, like using variable sizes of the patch, adding stubs of variable lengths and use of variable size slots on the ground plane. Further, techniques for circular polarization in space fed arrays are reviewed. They are achieved by rotating the patches at certain angles. Techniques for orthogonal polarization have also been reviewed. Towards the end of the paper recent and future applications for reflectarrays have been reviewed.
\end{abstract}

\section{Keywords}

Microstrip patch, Stub, Microstrip Reflectarray, Circular Polarization, Orthogonal Polarization

\section{INTRODUCTION}

The printed reflectarray antenna or flat reflector consists of a very thin, flat reflecting surface and an illuminating feed. On the reflecting surface, many printed microstrip patches or dipoles may be implemented without any power division network. The reflectarray feed illuminates the printed elements which in turn are designed to scatter or re-radiate the incident field with a planar phase front in a designated direction. The reflectarray is an antenna consisting of either a flat or a slightly curved reflecting surface and an illuminating feed antenna as shown in Fig 1. The radiating elements such as open - ended waveguides, printed microstrip patches, dipoles, or rings on the reflecting surface do not have any power division transmission lines. The feed antenna spatially illuminates these reflectarray elements that are predesigned to reradiate and scatter the incident field with electrical phases that are required to form a planar phase front in the far-field distance [1-2]. A major drawback of reflectarray antennas is that phase compensation needs to be done for the noncentered patches. The distance travelled by the radiated waves to the non-centered patches is more as compared to the center patch [1-2].

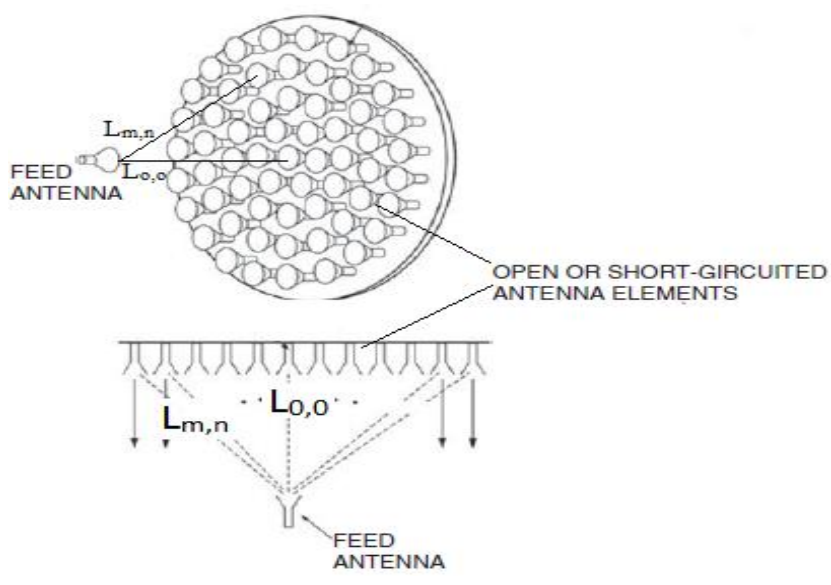

Fig 1: Configuration of reflectarray antenna in 2-D and 3-D view [1].

\section{REFLECTARRAY WITH VARIABLE SIZE PATCHES}

Microstrip patch elements are resonant structures, where the length of the patch decides the resonance frequency. A rectangular microstrip antenna (RMSA) is resonant when it has length, $\mathrm{L}=\lambda_{\mathrm{g}} / 2$, at the fundamental mode. Any deviation from this length detunes the element from its resonance frequency. By changing the length, a space - fed patch element with desired phase can be obtained along with his increased broadside directivity. The waves which are incident toward off- centered patches on the reflectarray travel an additional distance as compared to those incident at the central patch. Therefore, the waves which are re- radiated from the off-centered elements of the reflectarray will have different phase with respect to the phase of the waves which are re- radiated from the center element. In order to have the same phase of the re- radiated waves, the lengths of the non- central elements are shortened to detune with respect to the length of the center array element, thereby resulting in the uniform phase of the reflected wave front [3].

A $4 \times 4$ microstrip array, fabricated on a substrate, is shown in Figure 3. In order to have forward radiation from the elements to be constructive along $\theta=180^{\circ}$, the phases of the adjacent elements are required to be adjusted. Since the patch elements are fabricated on substrate, the effective resonant length is $\mathrm{L}=$ $\lambda_{\mathrm{g}} / 2$ for the element at point $\mathrm{P}$, which is right above the fed RMSA. Elements away from this point need to be detuned to obtain the necessary progressive phasing. The resonant length of the element that would be centered at point $\mathrm{P}$ is taken as reference for the required phasing. Considering the four elements around the point $\mathrm{P}$, there has to be phase adjustment in the form of resonant dimension adjustment of these elements. The length ' 1 ' and width ' $w$ ' of these elements are calculated as $1=(\mathrm{L} \times \mathrm{d}) / \mathrm{D}$ and $\mathrm{w}=(\mathrm{W} \times \mathrm{d}) / \mathrm{D}$, where $\mathrm{d}=$ 
$\left[\left(S_{x} / 2\right)^{2}+\left(S_{y} / 2\right)^{2}+D^{2}\right]^{1 / 2}, S_{x}$ and $S_{y}$ are the spacing between the centers of the adjacent elements in $\mathrm{x}$ and $\mathrm{y}$ direction respectively, $\mathrm{D}$ is the distance between point $\mathrm{P}$ and center of feed which is multiple of $\lambda_{\mathrm{o}} / 2$. Similarly, the dimensions of the next ring of patches are calculated taking account of their distances from the reference point $\mathrm{P}[3]$.

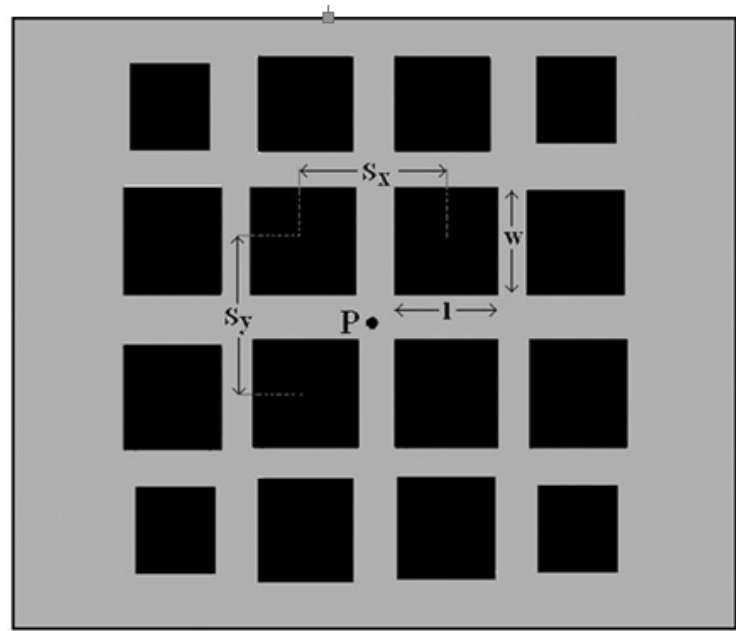

Fig 2: The schematic of reflectarray with variable patches [3]

Consider a reflectarray designed at $5.4 \mathrm{GHz}$, with a main beam scan angle of $30^{\circ}$ from the normal direction of an array. Fig. 4 shows the calculated phase of the total reflected field from an infinite patch array against the resonant length of the patch. This result shows that the patch lengths can be adjusted to give a total phase shift of about 330. Also shown are the phase against length curves for two other frequencies, demonstrating that the phase variation with patch size is uniform with a change in frequency [3].

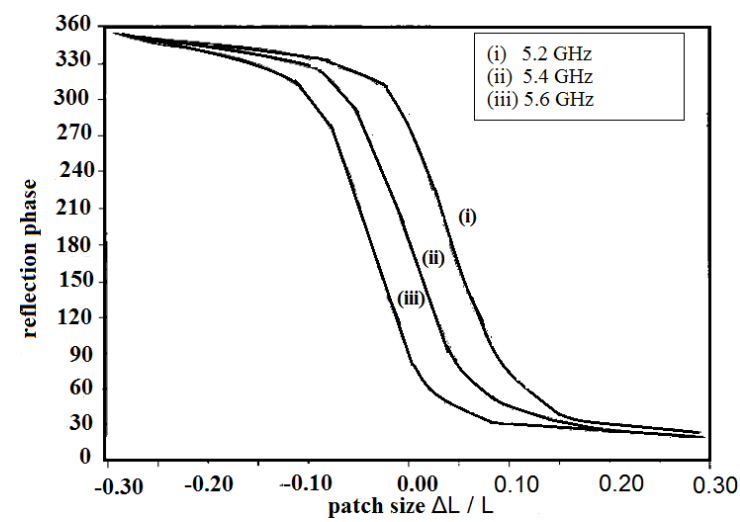

Fig 3: Calculated phase of the field reflected from on infinite array of microstrip patches against patch size [3]

\section{REFLECTARRAY WITH VARIABLE STUB LENGTHS}

The design and analysis of a reflect array utilizing a stub loaded microstrip element is shown in Figure.5(a).The reflection, scattering and total field matrices reduce to the coefficients $\mathrm{R}, \mathrm{S}$ and $\mathrm{R}^{\mathrm{tot}}$. Conservation of energy for a lossless dielectric slab requires $|R|=1,|R+\mathrm{s}|=1$ and $0<|S|<2$. At the element resonance the magnitude of $S$ equals two and is exactly $180^{\circ}$ out of phase with R. The calculated phase and amplitude of the total reflection coefficient for an infinite array of microstrip elements measuring $1.701 \mathrm{~cm}$ by $2.382 \mathrm{~cm}$ is shown in Figure. 5(b) as a function of stub length.
The stub length width is $0.046 \mathrm{~cm}$. The electrical length of the stub was varied from $0^{\circ}$ to $180^{\circ}$ at $5350 \mathrm{MHz}$. The substrate exhibited a relative dielectric constant of 2.3 and a thickness of $1.8 \mathrm{~cm}$. The antenna is designed to exhibit an unloaded resonant frequency of $5350 \mathrm{MHz}$ [4]

At $5350 \mathrm{MHz}$, the phase of the total reflection coefficient is nearly a linear function of stub length and that the phase of the reflection is nearly twice the electrical stub length. At frequencies $150 \mathrm{MHz}$ from the antenna resonant frequency the reflection coefficient phase becomes distorted. These curves have been used to design and analyse the performance of a CBand reflect array with excellent results [4].
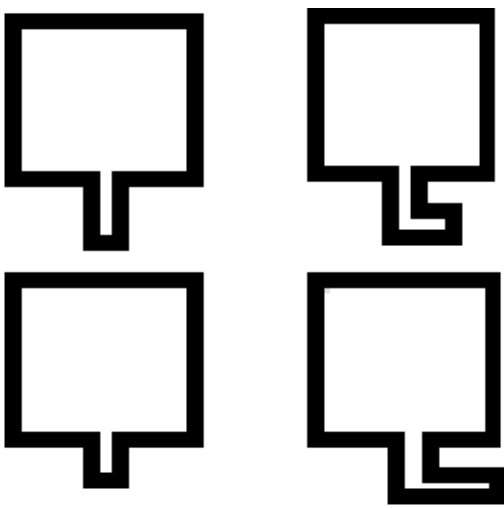

Fig 4: Patches with variable length phase delay lines [4]

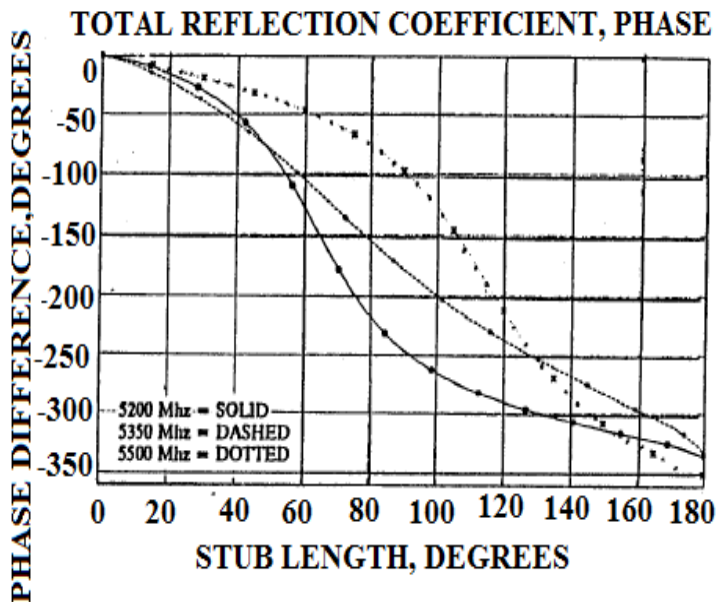

Fig 5: Phase of total reflection coefficient as a function of stub length [4]

\section{REFLECTARRAY WITH VARIABLE SIZE SLOTS ON GROUND PLANE}

A class of novel reflectarray antenna topologies is proposed in [5]. The common features of these multilayer printed reflectarray topologies are the presence of microstrip patches of similar size arranged into a regular lattice configuration on the top layer, and periodic configuration of slots of variable size on the lower layer that are utilised to adjust the phase shift of the incoming wave. Different topologies arise from the addition of a ground plane below the slot layer in order to reduce the back lobe radiation, and an additional substrate layer on top of slots for improvement of the bandwidth 


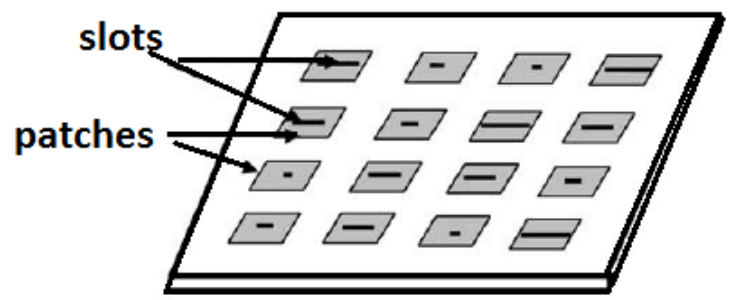

Fig 6: Top view of MRA with slots in ground plane [5]

4.1 Reflectarray with Slots in Ground Plane One novel reflectarray proposed in [5] is composed of radiating patch elements of identical size on the top layer and slots of variable length in the ground plane. The required phase shift at each position on the reflectarray surface is obtained by adjusting the slot length on the ground plane. Figure 6 and 7 show the top and side views of this configuration.

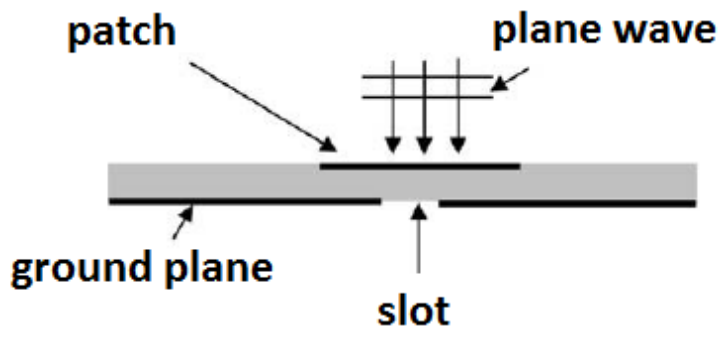

Fig 7: Side view of MRA with slots in ground plane [5]

The incident wave from the feed excites the dominant mode on the microstrip patches. When there is no slot on the ground plane, each patch radiates energy at its resonant frequency. The presence of slots acts as an inductive loading on the patches, which introduces a phase shift in the patch response. The inductance of each slot depends on its length [5].

\subsection{Reflectarray with Two Layers and Variable Slots in the Middle Plane}

The difference between this configuration and the previous one is the presence of a ground plane beneath the slot layer, which prevents leakage of power into the lower half space. Figure 8 shows a schematic view of this reflectarray. The thickness of the substrate (which is thinner than the operating wavelength), and the fact that different phase values are imparted by the slots to the incoming wave, preclude the possibility of guided wave excitation. Also, the low level of antenna radiation in the end fire direction verifies the absence of guided waves [5].

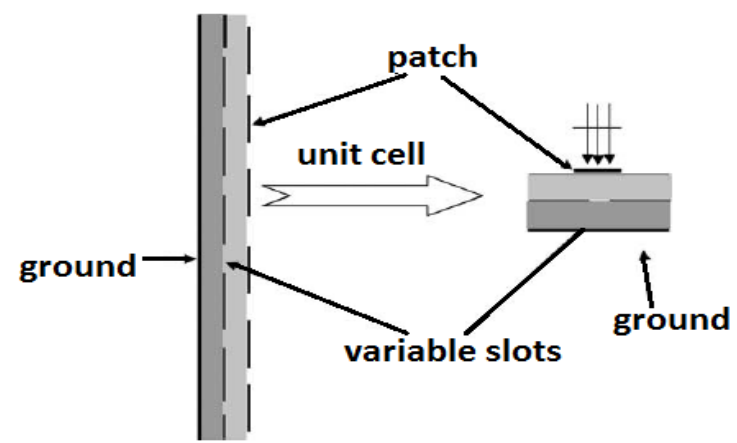

Fig 8: Reflectarray with two layers and variable slots in the middle plane [5]

\subsection{Reflectarray with Three Layers and Variable Slots in the Middle Plane}

The structure presented in this Section is the same as the twolayer reflectarray mentioned in the previous Section, except for an additional substrate layer between the variable slots and upper microstrip patch layer. This additional substrate layer improves the bandwidth and radiation pattern [5].

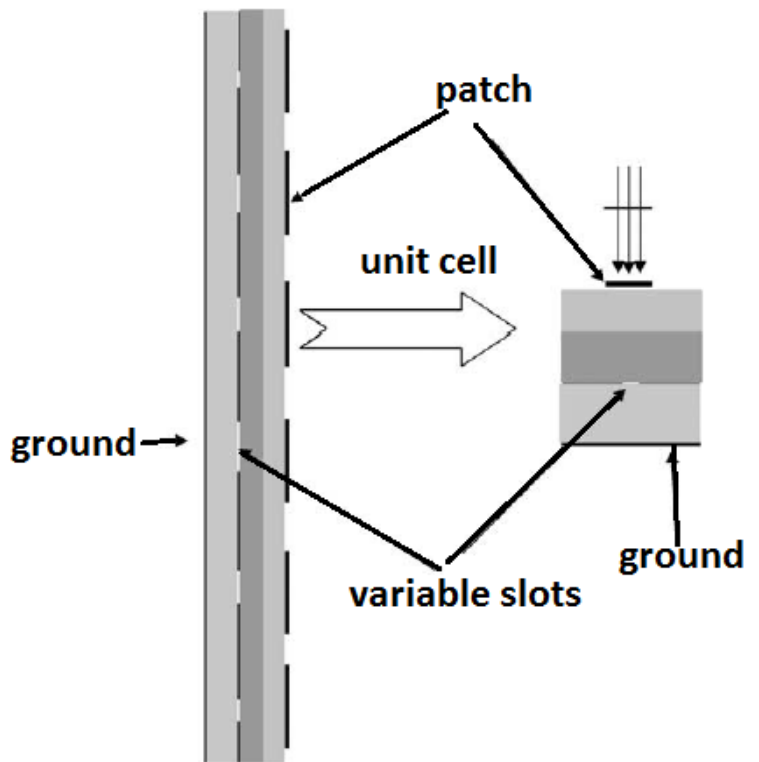

Fig 9: Reflectarray with three layers and variable slots in the middle plane [5]

\section{REFLECTARRAY WITH VARIABLE ROTATION ANGLES}

In [6] a circularly polarized microstrip reflectarray with elements having variable rotation angles is shown as a means of achieving co-phasal far-field radiation. Two microstrip reflectarrays have been designed. In the first one, square patches with variable-length microstrip phase-delay lines attached are used. The other one has identical square patches with identical microstrip phase-delay lines. In these patches the rotation angle of each element is different. Both antennas demonstrated excellent performance with more than 55\% aperture efficiencies, but the one with variable rotation angles resulted in better overall performance.

This paper [6] presents a new approach for a circularly polarized reflectarray to achieve a far-field beam by using identical microstrip patches having different angular rotations. A few elements of the reflectarray are shown in Fig. 7. It is known that if a circularly polarized antenna element is rotated from its original position by $\theta \mathrm{rad}$, the phase of the element will be either advanced or delayed (depending on the rotation direction) by the same $\theta$ rad. Here, small- and low profile printed microstrip elements are used in a reflectarray with continuously variable angular rotations to achieve far field phase coherence. When a miniature or micro machined motor is placed under each microstrip element, this microstrip reflectarray can be controlled to scan its main beam to different and wide angular directions. 


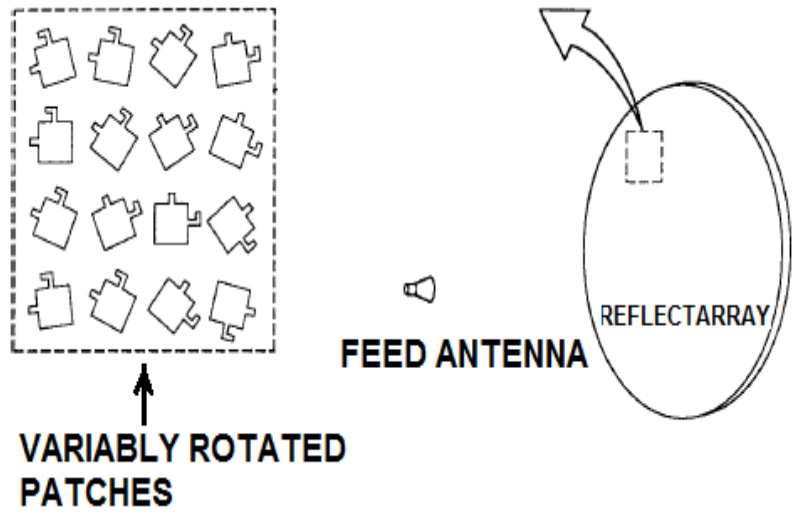

Fig 10: Circularly polarized microstrip reflectarray with elements having variable rotation angles [6]

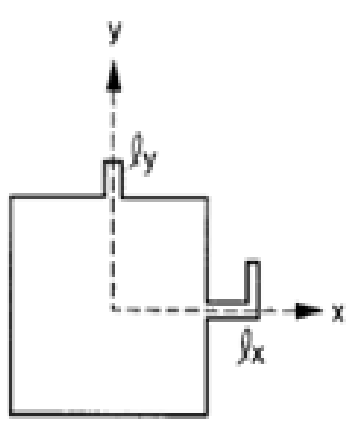

(a)

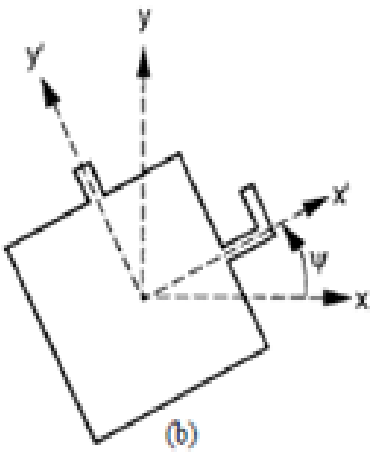

(b)
Fig 11: Circularly polarized reflectarray patch element. (a) Reference element with $0^{\circ}$ phase shift. (b) $\psi$ degree rotated element with $\psi / 2$ degree phase shift [6]

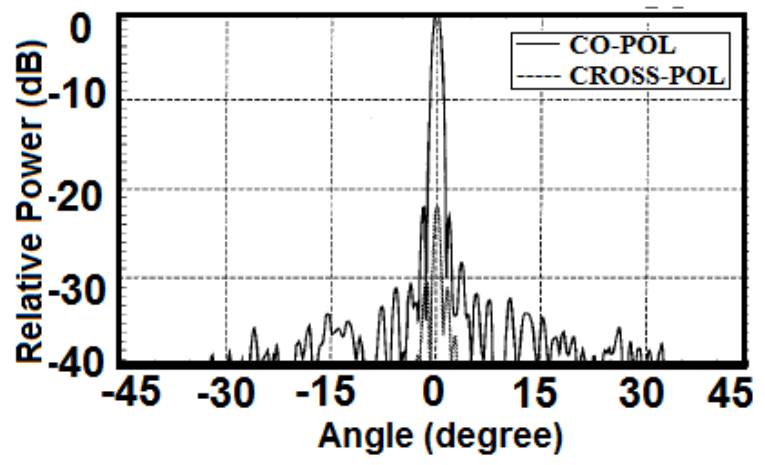

Fig 12: Measured radiation pattern of unit 1 reflectarray with patches having variable length phase-delay lines [6]

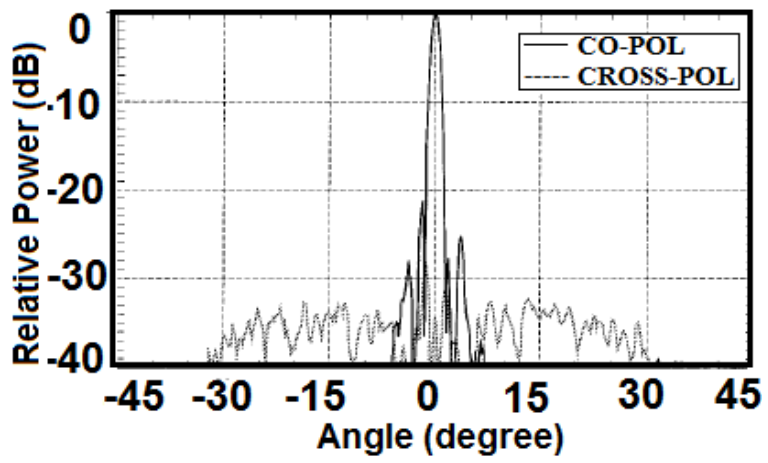

Fig 13: Measured radiation pattern of unit 2 reflectarray with patches having variable rotation angles [6]

\section{DUAL FREQUENCY MICROSTRIP REFLECTARRAY}

Microstrip antennas are known to have a narrow bandwidth. However, they exhibit many other advantages that make them suitable for mobile and satellite communications. While narrow in bandwidth, microstrip antennas are well-suited for dual-frequency operation. Recent developments in microstrip reflectarrays have further demonstrated the viability of printed-circuit technology for space communications [7]. Combining the novel characteristics of microstrip reflectarrays with the concept of dual-frequency operation, the feasibility of a stacked dual-frequency microstrip reflectarray has been examined. To achieve dual-frequency, consider a stacked configuration which was first suggested in [8]. Fig 11 shows an example of a sub-array geometry for the dual frequency reflectarray. In this configuration, the first layer antenna is for lower frequency operation while the upper layer antenna is for higher frequency operation. To simplify the design process, the upper layer sub-array patches should fall completely inside the perimeter of the lower layer patch thus minimizing the effect of the lower substrate. The conceptual design process for this stacked antenna consists of first designing the lower layer antenna by assuming a substratesuperstrate configuration while ignoring the presence of the upper layer antenna and designing the upper layer antenna by assuming a single layer configuration while ignoring the presence of the lower layer patch. This design process, in reality, yields only a first iteration design because the performance of the composite antenna will be affected by mutual coupling between the layers and higher-order modes generated by the lower layer antenna, particularly at high frequencies. The viability of a stacked microstrip reflectarray for dual frequency operation has been demonstrated. The proposed multilayer configuration is easy to manufacture by an etching process and opens new possibilities as an alternative to parabolic reflectors [7]

The stacked configuration opens new possibilities in the applications of microstrip reflectarrays. The design process showed that while the effect of the upper layer antenna is minimal at the lower frequency band, the presence of the lower layer antenna must be included in the design of the upper layer antenna [9].

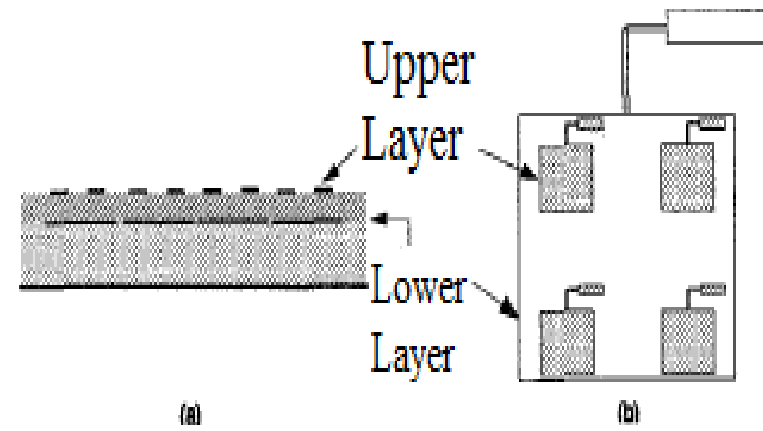

Fig 14: Configuration for a dual-frequency microstrip reflectarray. (a) Cross sectional view. (b) Top view of a section of reflectarray [9]

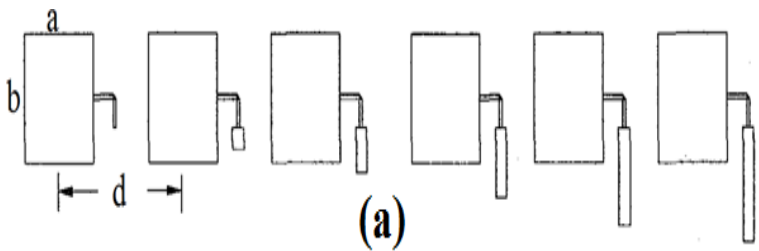


Incident Wave
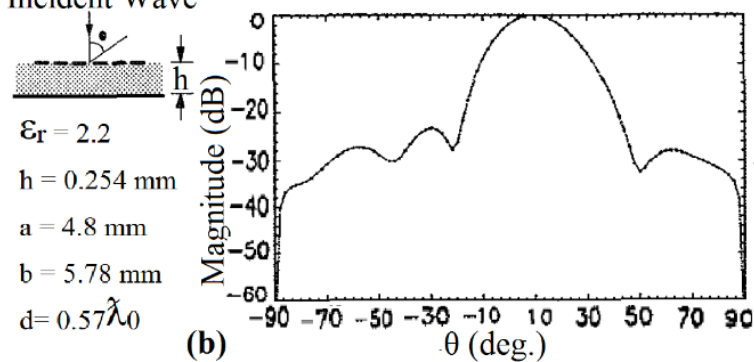

Fig 15: A section of microstrip reflectarray. (a) Geometry. (b) Normalized re-radiated field in the E-plane [9]

\section{DUAL POLARIZED MICROSTRIP REFLECTARRAY}

To be able to polarize the reflected signal in two planes requires that the array elements be polarized in both $\mathrm{x}$ - and $\mathrm{y}-$ directions. Square microstrip patch antennas can be used for this purpose. To excite both polarizations simultaneously, two feed-horns were used, one polarized in the x-direction and the other in the y-direction. For the phase-shifting stubs to be functional, they must be aligned with the polarization direction of the microstrip patches. Thus, the additional polarization in the y-direction requires that an additional microstrip stub be added to each element. The two stubs on each element must also operate independently of each other, and the required orthogonality can be achieved by placing the two stubs on perpendicular edges. An illustration of the array which was used is shown in Fig 13. The design of a square microstrip patch follows the same procedure as for a rectangular patch. The array will be designed on RT-Duroid 5870 substrate with $\varepsilon_{\mathrm{r}}=2.3$ and thickness of $0.381 \mathrm{~mm}$. The operating frequency is again $10.0 \mathrm{GHz}$ which gives the dimensions of the patch to be $W=L=9.56 \mathrm{~mm}$. The input impedance to this antenna at either edge is calculated, resulting in an input impedance of 188 ohms. A microstrip line with the identical characteristic impedance has a width of $0.05 \mathrm{~mm}$ which is difficult to realize. Thus, our stubs cannot be perfectly matched to the patch antennas. Using the same $130 \mathrm{ohm}$ stubs gives a voltage standing wave ratio (VSWR) of 1.44 which is small enough to give us satisfactory results. The array was designed to have a scan angle of 20 degrees with an element spacing of $0.6 \lambda_{0}(18 \mathrm{~mm})$ in both the $\mathrm{x}$ and $\mathrm{y}-$ directions. This requires a progressive phase shift of -73.88 degrees which must be realized in both $\mathrm{x}$ - and $\mathrm{y}$ directions [10].

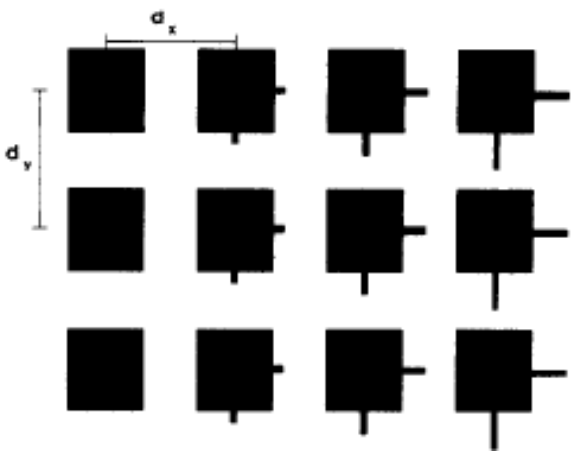

Fig 16: Dual-polarized reflectarray [10]

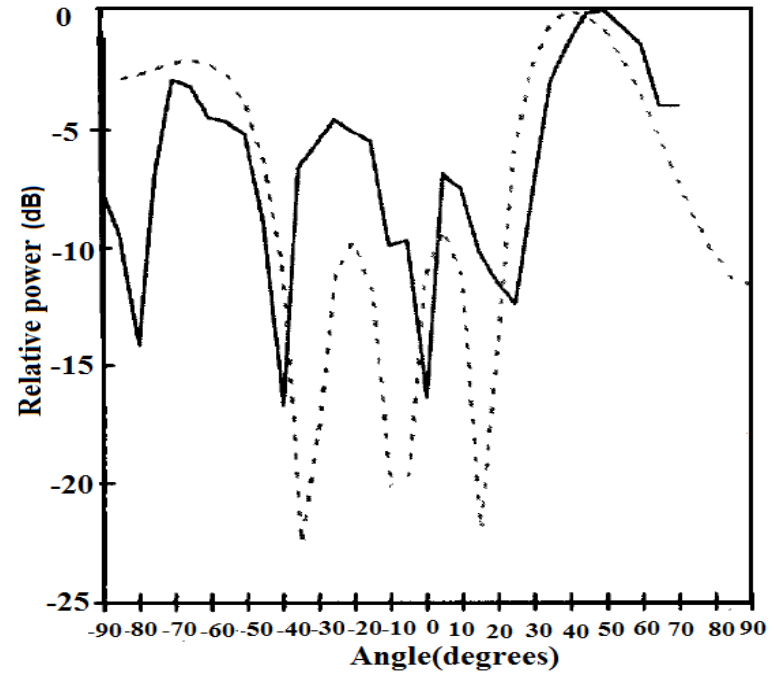

Fig 17: E-Plane redirected radiation pattern with the incident wave at 20 degrees [10]

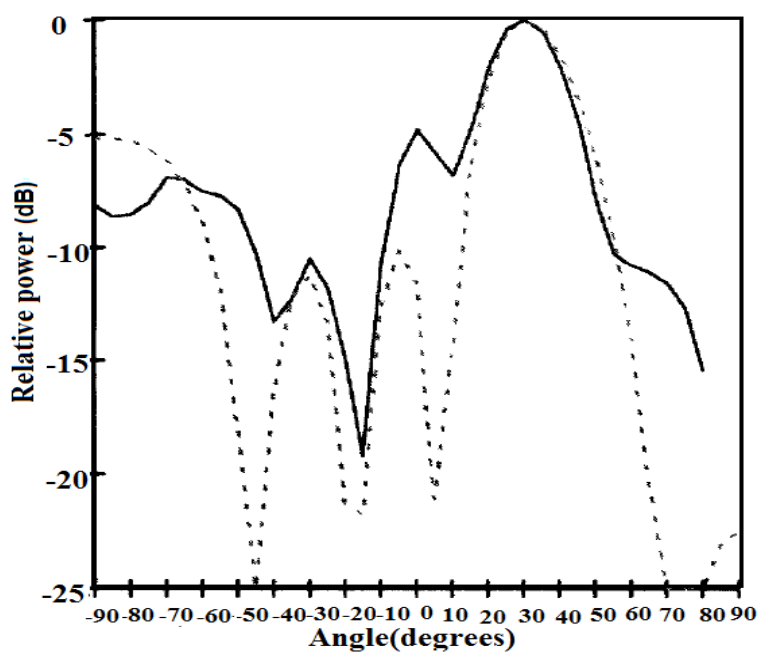

Fig 18: E-Plane redirected radiation pattern with the incident wave at 10 degrees [10]

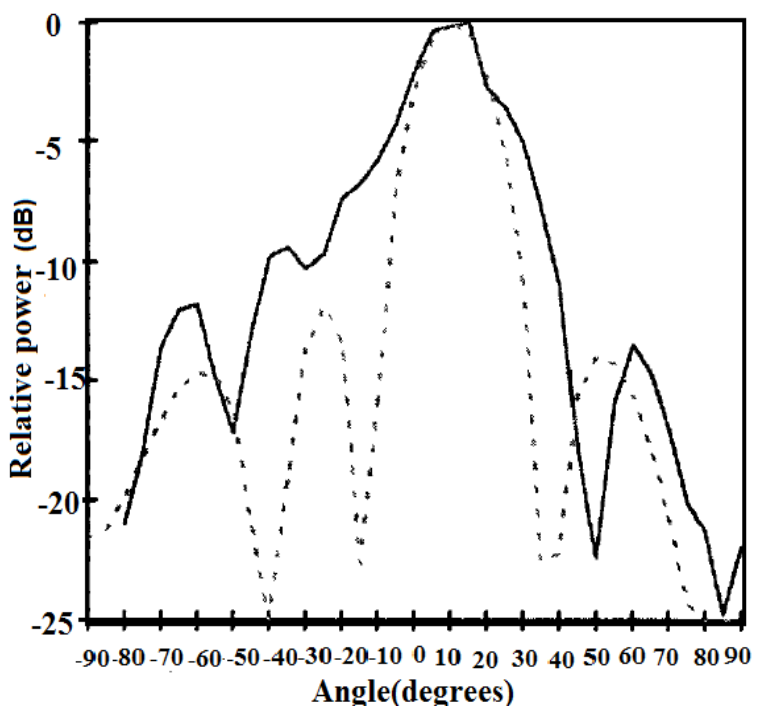

Fig 19: E-Plane redirected radiation pattern with the incident wave at $\mathbf{- 1 0}$ degrees [10] 


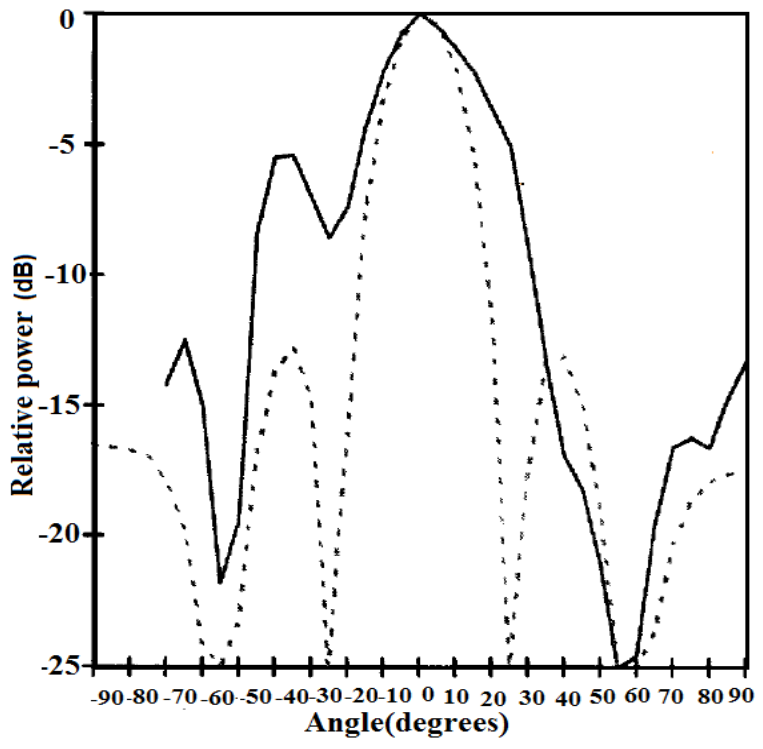

Fig 20: E-Plane redirected radiation pattern with the incident wave at $\mathbf{- 2 0}$ degrees [10]

\section{RECENT AND FUTUTRE APPLICATIONS}

\subsection{Multi-Beam Reflectarrays}

Reflectarrays can be designed to generate multiple beams, either with a single feed, or with one or more feeds per beam. In the latter case, the antenna configuration and the design process is similar as in multi - feed reflectors. However, the flexibility provided by reflectarrays to achieve any value of phase - shift independently for each linear polarization, can be used to improve the performances in multi-beam applications, to produce various simultaneous shaped beams, and to achieve several beams with a single feed.

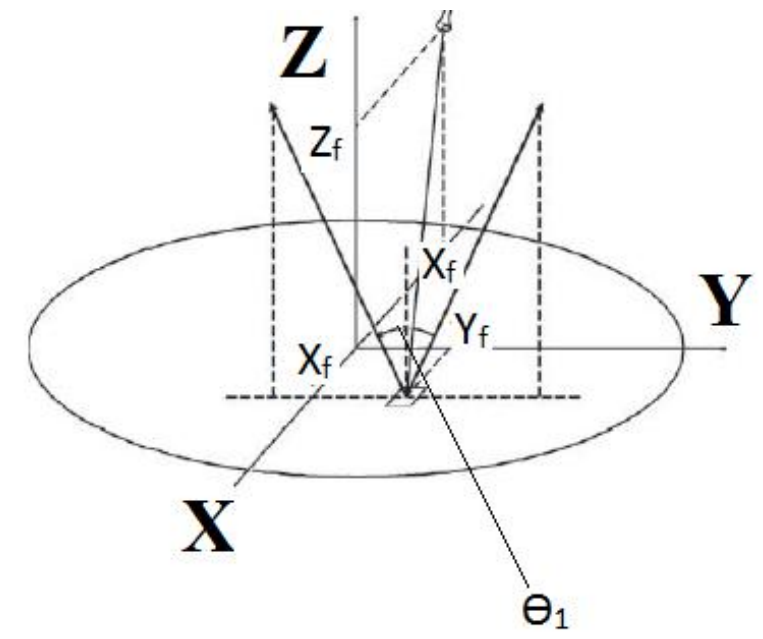

Fig 21: Antenna geometry of two-beam reflectarray [1]

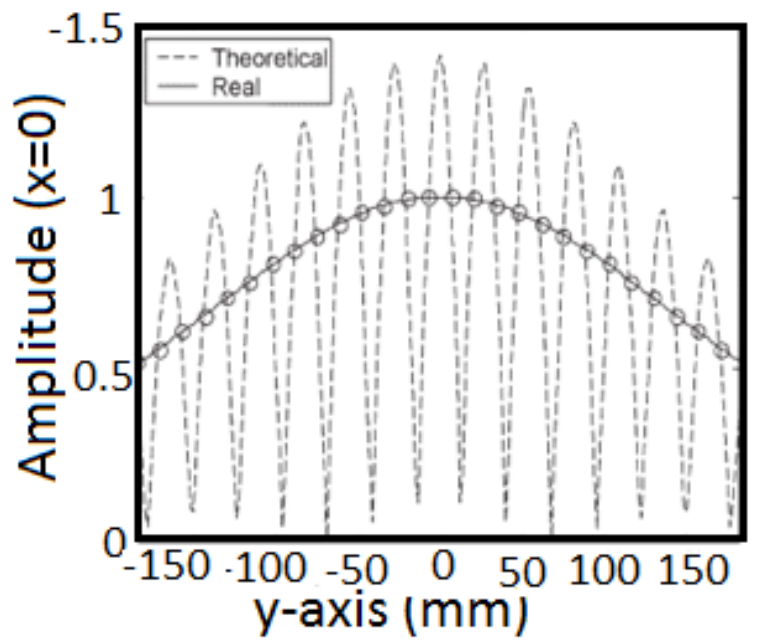

Fig 22: Amplitude distribution in y-direction of two-beam reflectarray [1]

\subsection{Amplifying Reflectarray:}

The active devices have been integrated with reflectarray antennas to provide amplification, however these antennas do not possess phase changing ability. Recently, an amplifying reconfigurable reflectarray element is demonstrated to achieve an overall gain of $12 \mathrm{~dB}$ with a $360^{\circ}$ phase agility at $5.8 \mathrm{GHz}$ [11]. The reflectarray element consists of an aperture-coupled microstrip patch that is capable of receiving a linearly polarized incident wave and re-radiating an orthogonally polarized wave. The feed array, shown in Figure 14, has four microstrip patches with both transmit and receive capabilities. The vertically polarized transmit feed array sends signals to the reflectarray elements, where they get amplified, their polarization is changed to horizontal, and then reradiated to focus the beam to the horizontally polarized receive array. A reflection-type phase shifter (RTPS) and an amplifier circuit will phase- shifts and amplify the incident signal coupled via slot 1 , later couples it to the orthogonal polarization via slot 2 . The required phase of each element for the reflectarray to radiate a co - phasal plane wave is achieved by varying the length of the transmission line between the two orthogonal ports.

The patch, slot, stub and offset dimensions are optimized to achieve impedance matching of $50 \Omega$. The slots are offset from the center of the patch in the $\mathrm{x}$ direction only to maximize the isolation between the signals on the input and output transmission lines, to maintain stability. Since the presence of the active device and feedback paths can result in oscillations stability of amplifier is necessary.Thus, through the above process, the signal gets amplified and spatially combined without encounter any significant loss. This power combiner achieved a power gain of about $7 \mathrm{~dB}$ along with providing 362 degree of phase tunability and a maximum amplification gain of $11.3 \mathrm{~dB}$. 


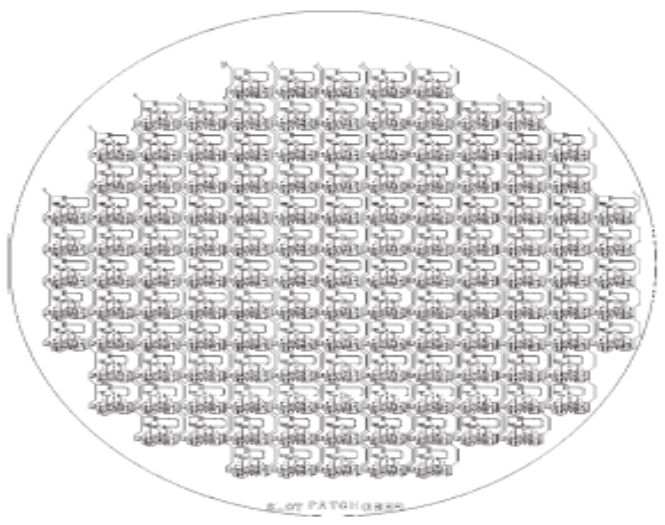

Fig 23: 137-element microstrip amplifying reflectarray [1]

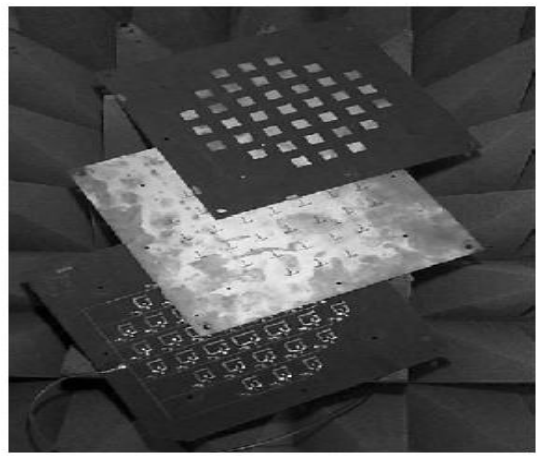

Fig 24: Photograph showing three layers (patch, slot, and amplifier) of microstrip amplifying reflectarray [1]

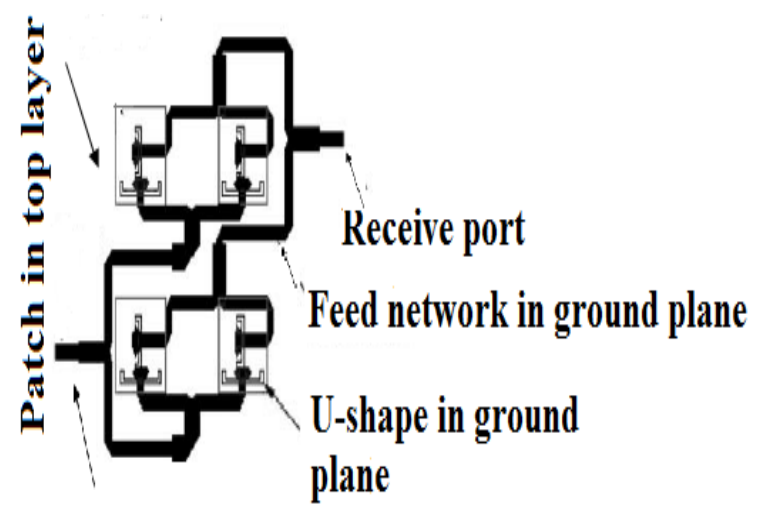

Transmit port

Fig 25: Feed array with four patches that transmit vertical polarization while receive horizontal polarization [1]

\subsection{Folded Compact Reflectarray:}

In reflectarray, as in reflector antennas, the feed is placed at a certain distance from the $\alpha_{\text {feed }}$ reflecting surface, removing the low - profile characteristic of printed antennas. In order to overcome this problem, a more compact configuration called a "folded reflectarray antenna" has been proposed for linear polarization applications [12-13]. The folded configuration consists of a printed reflectarray with a feed embedded in its structure and a polarizing grid placed parallel to the reflectarray at a certain distance h, as shown in Figure.17 (a) $\&$ (b). The polarizing grid is made of a strip grating printed on a dielectric slab, so that it behaves as a reflector for the electric fields parallel to the grating while it is transparent for the orthogonal polarization. Alternatively, the grating can be substituted by a slot - array. The folded configuration allows a reduction of the antenna depth to approximately one half of the focal distance.

The feed produces an approximately linearly polarized spherical wave with the electric field parallel to the grating, which is reflected by the polarizing grid towards the printed reflectarray. The reflectarray is formed by variable size patches rotated by $45^{\circ}$ with respect to the direction of incident electric field. The reflectarray is designed to properly focus or shape the beam and to twist the polarization by $90^{\circ}$ with respect to the incident wave, so that the radiated field can pass through the polarizer. The polarization rotation is produced by adjusting the patch dimensions to cause a phase difference of $180^{\circ}$ between the reflection phases of the two incident wave components, as schematically shown in Figure 18. The polarizing redone is designed with the grating printed on a dielectric substrate, whose thickness is approximately half a wavelength, to allow the field radiated by the reflectarray to pass through with minimum insertion losses [1].

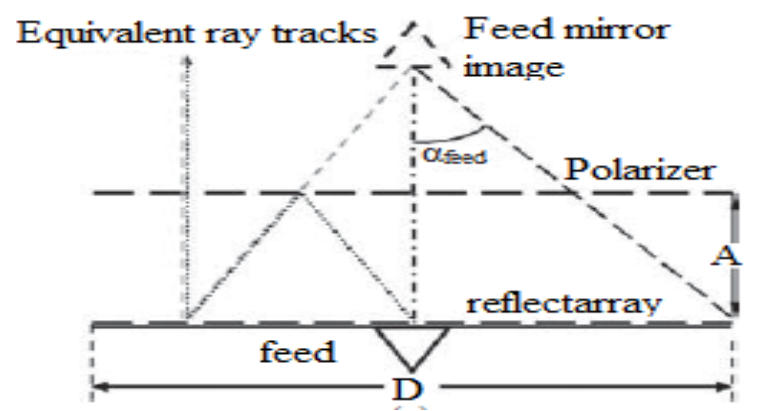

Fig 26: Lateral view with the equivalent feed of folded printed reflectarray [1]

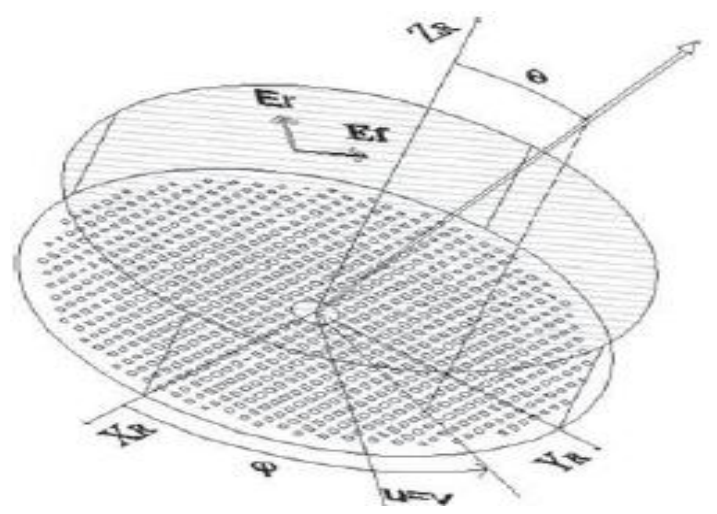

Fig 27: 3-D view of Folded printed reflectarray [1]
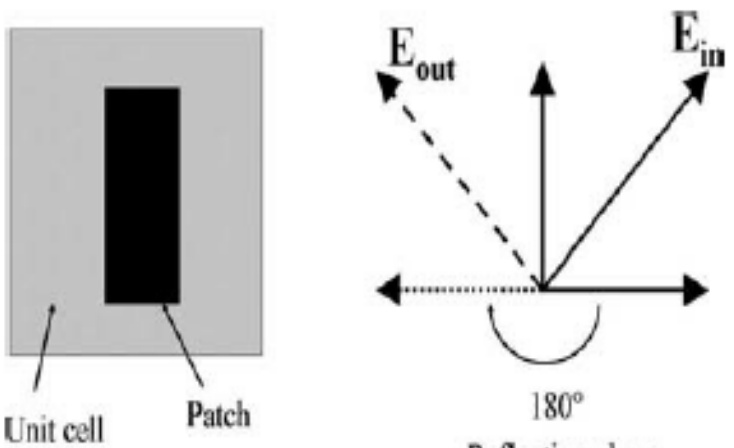

$180^{\circ}$

Reflection phase

Fig 28: Illustration of the orthogonal components of the incident and reflected electric field for a difference of 180 in the reflection phases [1] 


\section{CONCLUSION}

Relflectarrays use an array of elements that provide preadjusted phasing to form a focused beam when it is illuminated by a feed. The feeding mechanism eliminates the complexity and losses of the feeding network used in planar arrays, thus providing a higher efficiency. The use of variable size stubs and patches helps in compensating the phase difference between the elements. Phase compensation for a large number of elements, orthogonal and circular polarization and the effects of varying various parameters will be discussed in detail in further research.

\section{REFERENCES}

[1] Huang, J. and Encinar, J. Reflectarray Antennas, John Wiley 7 Sons, Inc.

[2] Huang, J. 1996 Capabilities of printed reflectarray antennas, IEEE symposium on Phased Array System and Technology, Boston, Massachusetts, October 1996, pp. $131-134$

[3] Pozar D.M. and Metzler T.A., 1993, Analysis of a reflectarray antenna using microstrip patches of variable size, Electronics Letters, Vol. 29, no. 8, pp. 657- 8, 1993.

[4] Metzler, T.A. 1995 Stubloaded microstrip reflectarrays, IEEE.

[5] Chaharmir M.R., Shaker J., Cuhaci M. and Sebak A. 2003 Reflectarray with variable slots on ground planeIEE Proc.-Microw. Antennas Propag., Vol. 150, No. 6, December 2003.

[6] Huang, J. and Pogorzelski R.J. A Ka-band microstrip reflectarray with elements having variable rotation angles, IEEE Trans. Antennas and Propagation, Vol. 46, pp. 650 - 656, May 1998.

[7] Huang J., Wu D.I. and Hall R.C. 1995 Dual-frequency Microstrip reflectarray, IEEE AP - S/URSI symposium, 1995 , pp. $2128-2131$.

[8] Encinar J.A. Design of dual frequency reflectarray using microstrip stacked patches of variable size, Electronic Letters, Vol. 32 , No. 12 , June 1996 pp. 1049 - 1050.

[9] Huang J. 1991 Microstrip reflectarray, IEEE Intem. APSAJRSI Symp. Digest pp. 612-615, 1991.

[10] Javor R.D., Wu X. and Chang K., Design and performance of a microstrip reflectarray antenna, IEEE Transactions On Antennas And Propagation, Vol. 43, No. 9, September 1995.

[11] Bialkowski M.E., Robinson A. W. , and Song H. J., 2002, Design, Development, and testing of X - band amplifying reflectarrays, IEEE Trans. Antennas Propag. , Vol. 50, pp. 1065 - 1076, August 2002.

[12] Pilz D. and Menzel W. 1998 Folded reflectarray antenna, Electronics Letters, pp. 832 - 833, April 1998.

[13] Pilz D., Menzel W. and Leberer R. 1999, A 77 - GHz $\mathrm{FM} / \mathrm{CW}$ radar front - end with a low - profile low - loss printed antenna, I EEE Trans. Microwave Theory Tech. , Vol. 47 , No. 12 , pp. 2237 - 2241, Dec. 1999.

[14] Pilz D., Menzel W. and Al-Tikriti M. 2002, Millimeter wave folded reflector antenna with high gain, low loss, and low profile," IEEE AP Magazine, pp. 24 - 29, June 2002. 